SHORT REPORT

\title{
Serious neurological disorders in children with chronic headache
}

\author{
I Abu-Arafeh, S Macleod
}

Arch Dis Child 2005;90:937-940. doi: 10.1136/adc.2004.067256

\begin{abstract}
Aims: To determine the prevalence of serious neurological disorders among children with chronic headache.

Methods: All children presenting to a specialist headache clinic over seven years with headache as their main complaint were assessed by clinical history, physical and neurological examination, neuroimaging where indicated, and by follow up using prospective headache diaries. Results: A total of 815 children and adolescents (1.2518.75 years of age, mean 10.8 years (SD 2.9); 432 male) were assessed. Mean duration of headache was 21.2 months (SD 21.2). Neuroimaging (brain CT or MRI) was carried out on 142 (17.5\%) children. The vast majority of patients had idiopathic headache (migraine, tension, or unclassified headaches). Fifty one children (6.3\%) had other chronic neurological disorders that were unrelated to the headache. The headache in three children $10.37 \%, 95 \% \mathrm{Cl}$ $0.08 \%$ to $1.1 \%$ ) was related to active intracranial pathology which was predictable on clinical findings in two children but was unexpected until a later stage in one child $10.12 \%, 95 \%$ Cl $0.006 \%$ to $0.68 \%$ ).

Conclusions: Chronic headache in childhood is rarely due to serious intracranial pathology. Careful history and thorough clinical examination will identify most patients with serious underlying brain abnormalities. Change in headache symptomatology or personality change should lower the threshold for imaging.
\end{abstract}

$\mathrm{H}$ eadache is a common problem in childhood; up to $25 \%$ of schoolchildren suffer from chronic recurrent headache. Most headaches are idiopathic in nature; at least $10 \%$ of children between 5 and 15 years of age suffer from migraine. ${ }^{1}$ Secondary headaches, on the other hand, are rare and the incidence of brain tumours in children is 3-5 per 100000 (up to 1 in 20000 ). ${ }^{2}$ Around 1 in 10 of children with brain tumours (up to 1 in 200000 ) present with headache as their only symptom and have a normal physical examination leading to a delayed diagnosis. ${ }^{3}$ Therefore, for every child with brain tumours, there are around 5000 children with recurrent headache, including up to 2000 children with migraine. Furthermore, for every child with a brain tumour, presenting with headache as the only feature, there are at least 50000 children with recurrent headache, including up to 20000 with migraine. However, brain tumour and sinister intracranial disorders are of significant concerns to physicians, patients, and their families.

Several studies to assess the value of neuroimaging in the assessment of all patients presenting with headache were reported in the literature but failed to show any benefit. In a prospective randomised trial of children attending a paediatric neurology clinic with headache, patients either underwent brain magnetic resonance imaging (MRI) or were given a clinical follow up. Seventeen of 96 children had abnormal neuroimaging, but none had an abnormality that would require neurosurgical intervention. ${ }^{4}$ In a study of children attending the emergency room with headache, nine of the 150 children who were investigated with neuroimaging had surgically treatable conditions, but in all nine patients the abnormalities were predictable on clinical assessment and no child had a brain tumour unexpectedly. ${ }^{5}$ Similar retrospective studies have shown similar results. ${ }^{6}{ }^{7}$ Therefore, investigating all children with chronic headache using cranial MRI or computed tomography (CT) scan is not a feasible option, despite the anxiety that chronic headache may provoke to both carers and doctors. ${ }^{8}$ Neuroimaging is, nevertheless, a useful investigation for the population at high risk. Our clinical indications for neuroimaging of children with chronic headache are summarised in box $1 .{ }^{9}$

The purpose of this study is to assess the prevalence of expected and unexpected serious neurological disorders in children presenting with headache as their only complaint to a specialist clinic, and to identify any salient features that may increase the sensitivity of the list of indications for neuroimaging.

\section{METHODS}

All patients attending our headache clinic between 1996 and 2003 were eligible for inclusion in the study. Demographic and clinical information were collected prospectively on each child at every clinic appointment. Data included child's age, sex, duration of illness, frequency and duration of headache attacks, diagnosis, investigation, treatment, and also follow up arrangement. The diagnoses of different types of headache were made on the fulfilment of the International Headache Society's diagnostic criteria 1988. ${ }^{10}$ Neuroimaging and any other investigations were carried out as clinically indicated only. Children were followed up for as long as clinically necessary. The Fraser of Allander Neurosciences Unit is the only paediatric neurology facility in the region and we are not aware of any of the clinic patients presenting with serious intracranial disorders to other services.

Data were stored and statistically analysed on SPSS version 9.0 for Windows program and Confidence Interval Analysis. ${ }^{11}$ The children and parents of the three cases that are presented have given informed consent for publication. Our hospital's ethics committee accepted this study as an audit of clinic experience and, therefore, an application for ethical approval was considered unnecessary.

\section{RESULTS}

A total of 815 patients (432 male) attended the headache clinic over the seven year period. Mean age at assessment was 10.8 years (range 1.25-18.75, SD 2.9). Mean duration of headache was 21.2 months (range 1-144 months, SD 21.2). The majority of children were referred to the clinic by their general practitioners $(493 ; 60.5 \%)$, and the 322 children 
Box 1: Indications for neuroimaging in children with headache?

- Features of cerebellar dysfuction

- Ataxia

- Nystagmus

- Intention tremor

- Features of increased intracranial pressure

- Papilloedma

- Night or early morning vomiting

- Large head

- Focal new neurological deficits

- Recent squint

- Focal seizures

- Personality change

- Deterioration of school work

(39.5\%) were tertiary referrals from general paediatricians, paediatric neurologists, ophthalmologists, and ENT surgeons.

Neuroimaging in the form of brain CT scans, MRI, or both was carried out on $142(17.5 \%)$ children (fig 1). On the application of our clinical criteria for neuroimaging, 30 of the 478 children, who were referred by general practitioners with no previous investigations $(6.3 \%, 95 \%$ CI $4.3 \%$ to $8.8 \%)$, were scanned. In comparison, 74 children of the 322 who were referred by specialist paediatricians had brain MRI or CT scan before referral $(23 \%, 95 \%$ CI $18.4 \%$ to $27.6 \%)$.

The causes of headache are presented in table 1. Unrelated chronic neurological disorders were present in 51 children $(6.3 \%)$ : epilepsy $(n=29)$, cerebral palsy $(n=15)$, past history of encephalitis or meningitis $(n=4)$, and past history of craniopharyngioma $(\mathrm{n}=3)$.

The headache in three children $(0.37 \%, 95 \%$ CI $0.08 \%$ to $1.1 \%)$ was related to active intracranial pathology, two patients had brain tumours, and one patient had saggital sinus thrombosis. The intracranial pathology was predictable on clinical findings in two children (patient 1 and patient 3), but in one child (patient 2) it was unexpected during most of her headache history $(0.12 \%, 95 \%$ CI $0.006 \%$ to $0.68 \%)$. Clinical details of the three cases are presented.

\section{Patient 1}

A 6 year old boy presented with a three month history of daily headaches. Each attack lasted 1-3 hours. Pain was described as throbbing in nature; it was severe enough to stop some activities and often wakened him at night. During attacks he complained of vomiting, photophobia, and phonophobia. His parents noticed that he was squinting on watching television. On examination, he was clumsy, with nystagmus on looking to the left, diplopia on looking to the right, and papilloedema. An urgent CT of the brain showed a mass lesion in the posterior fossa with obstructive hydrocephalus.

\section{Patient 2}

A 7 year old girl was referred by her general practitioner with a six month history of headaches. She described throbbing headaches that occurred two or three times per month, lasting less than an hour and with no identifiable trigger factors. During episodes she was pale, complained of noise and light intolerance, and vomited in some attacks. She was well between the headaches, and examination, including fundoscopy was normal. A diagnosis of migraine without aura was made and she was asked to complete headache diaries. She failed to attend two subsequent follow up appointments. Two and half years later, she represented to the accident and emergency department with a four month history of an increasing headache frequency. New headache diaries revealed two distinct headache types, migraine without aura, and tension type headache. Neurological examination was still normal. Ibuprofen was suggested for her tension type headaches and she was reviewed again in six weeks. The migrainous headaches continued but tension headaches had abated. Migraine prophylaxis in the form of pizotifen was ineffective, but sumatriptan nasal spray was tried at age 9.9 years with an excellent response. A routine review appointment was brought forward at age 10.6 years. She was now complaining of daily brief headaches, usually in the early morning and sometimes associated with vomiting. She also complained of intermittent blurred vision, numbness at her lower back, and a loss of appetite. In view of her changing symptomatology an MRI brain scan was arranged which showed a left cerebellar hemisphere tumour with obstructive hydrocephalus. At operation the tumour was completely excised. Histopathology revealed a pilocytic astrocytoma.

\section{Patient 3}

This 14.5 year old girl was receiving treatment for nonHodgkin's lymphoma, including intrathecal methotrexate. She complained of daily, constant headache of moderate to severe intensity. No trigger factors were identified. Examination was unremarkable. Following an unsuccessful six week trial of aspirin an MRI brain scan was ordered. This showed a saggital sinus thrombosis, a recognised complication of intrathecal methotrexate. She was treated with heparin and acetazolamide. She had a good response with complete resolution of symptoms.

\section{DISCUSSION}

Population based studies of the prevalence of serious neurological disorders in children with chronic or recurrent headache are difficult to carry out for a number of reasons. Firstly, brain tumours in childhood are rare (3-5 per 100000$)$ and only 1 in 10 will present with headache as the only feature. Secondly, a clinic population of children with chronic headache is not an ideal representative sample of all children with headache, and it is reasonable to suggest that children attending a specialist headache clinic may reflect the severe end of the headache spectrum or have special concerns regarding the causes or management of their headache. While keeping these reservations in mind, we believe that this study provides an estimate of the highest possible proportion of children with severe neurological disorders that are likely to be identified in the population of children with chronic headache.

Serious causes of headache, such as brain tumours, are a real worry to parents and doctors. Brain MRI scans are safe and effective in the process of excluding the diagnosis of brain tumour. However, MRI scanning is neither readily accessible nor cost effective to be used in mass screening for patients with headache with no focal neurological deficits. ${ }^{12}$ CT scanning on the other hand is more accessible, but involves exposure to significant doses of radiation that makes it unsuitable for mass screening. Our data suggests that up to 1 in $4(23 \%)$ of the 322 children with chronic headache who were referred by specialist paediatricians have had a brain CT or MRI scan before referral to this clinic. It is not possible to comment on what were the indications for these investigations. In contrast, only a small number of children (53 of 726 


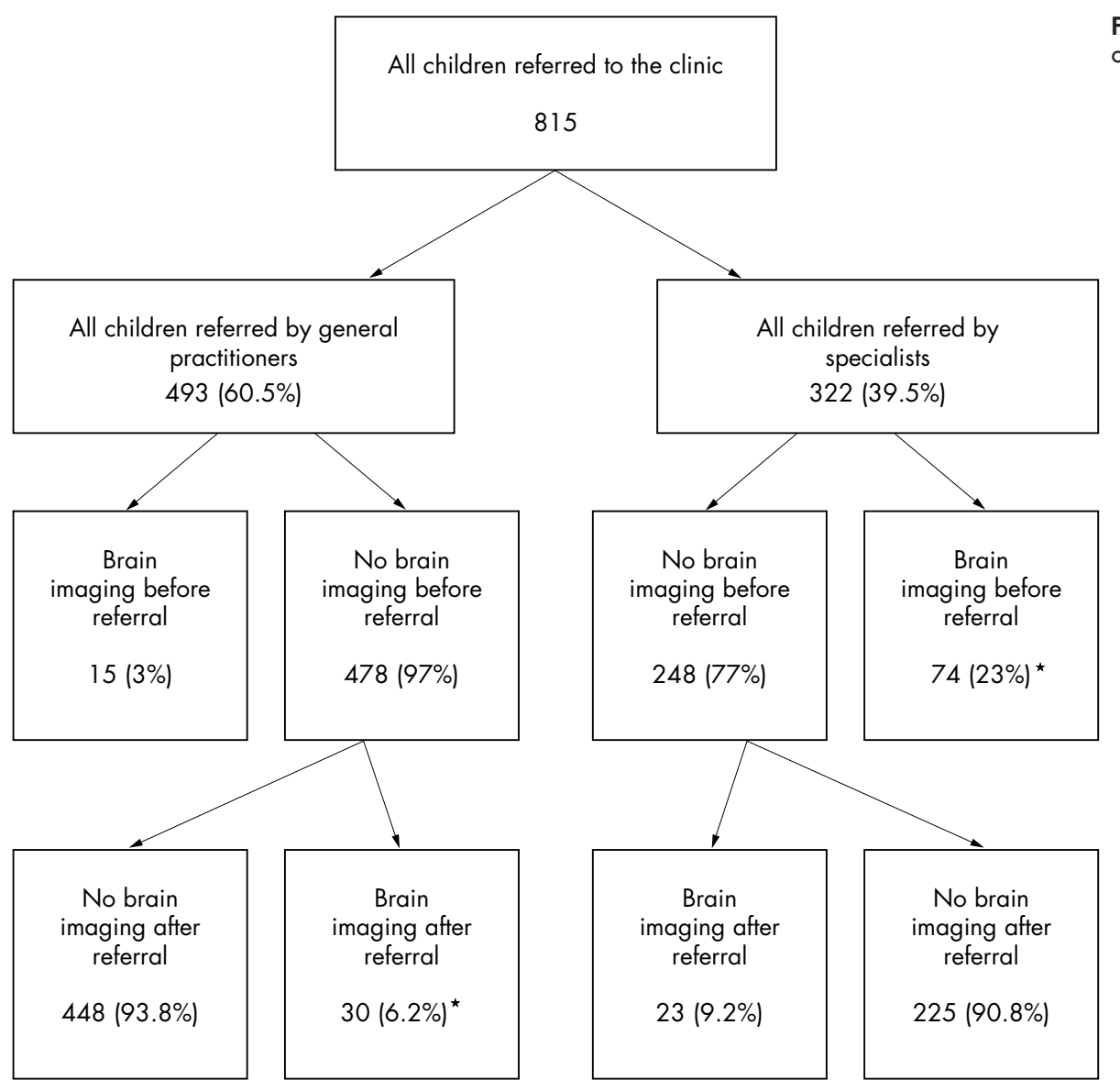

Figure 1 Clinic population and rates of neuroimaging.

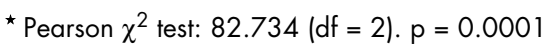

children; 7.3\%) were investigated with either a CT or MRI scan in the course of their assessment in this clinic and for clear indications, mainly for atypical headaches, hemiplegic migraine, or suspected intracranial lesions.

Serious neurological disorders in children with chronic headache are rare. Only three children in our study population (3.7/1000) had significant intracranial causes of the headache and required specific treatment. In one child (case 2) no neurological abnormality was expected for several years into her headache (1.2/1000). However, neuroimaging was requested when new symptoms evolved (early morning daily headaches, associated vomiting, intermittent blurred vision, numbness at her lower back, and a loss of appetite), raising the suspicion of a secondary headache. At this stage, it

Table 1 Causes of headache in clinic population

\begin{tabular}{lrr}
\hline Diagnosis & No. & \multicolumn{1}{l}{$\%$} \\
\hline Migraine without aura & 227 & 27.9 \\
Migraine with aura & 39 & 4.8 \\
Hemiplegic migraine & 15 & 1.8 \\
Episodic tension headache & 128 & 21.8 \\
Chronic tension headache & 178 & 21.8 \\
Mixed migraine and tension headache & 87 & 10.6 \\
Cluster headache & 1 & 0.1 \\
Unclassified headaches & 97 & 11.9 \\
Others & 43 & 5.3 \\
& & \\
Total & 815 & \\
\hline
\end{tabular}

is reasonable to consider the case as an expected abnormality, thus reducing the prevalence of unexpected serious neurological disorders to zero. However, the lack of symptoms of a brain tumour in this child for at least three years has highlighted several important issues:

- The changing pattern of headache, fluctuating between tension headache, migraine, and non-specific symptoms should lower the threshold for neuroimaging. Investigations of adult patients with chronic headache and normal physical examination have shown that atypical headaches are associated with increased risk of finding MRI abnormalities (14.1\%) compared to those with migraine $(0.6 \%)$ or tension headache $(1.4 \%){ }^{13}$

- Salient features of personality and emotional changes are to be taken seriously in the course of headache assessment. In a study of the causes of delayed diagnosis of brain tumours in children, it was noted that the nonspecificity of symptoms and the high incidence of psychological symptoms may have confounded the clinical picture and contributed to late diagnosis. ${ }^{14}$

- In patient 2 , it is likely that the brain tumour has developed on the background of chronic headache. Children with idiopathic headache have the same population standard risk for brain tumour and should be reviewed and assessed regardless of the previous illness or diagnosis.

- It is also likely that brain tumours presenting with headache over a long period of time without other features are benign in nature and slow growing. 


\section{Conclusions}

This study illustrates that chronic headache in childhood is rarely, if ever, due to serious intracranial pathology. Careful history taking and thorough clinical examination will identify patients with serious underlying brain abnormalities. A change in headache symptomatology or personality should lower the threshold for imaging. However, there is no role for routine neuroimaging in the management of children with idiopathic chronic headache.

\section{Authors' affiliations}

I Abu-Arafeh, S Macleod, Royal Hospital for Sick Children, Glasgow, UK

Competing interests: none

Correspondence to: Dr I Abu-Arafeh, Department of Paediatrics, Stirling Royal Infirmary, Livilands, Stirling FK8 2AU, UK; ishaq.abu-arafeh@ frah.scot.nhs.uk

Accepted 17 May 2005

\section{REFERENCES}

1 Abu-Arafeh I, Russell G. Prevalence of headache and migraine in schoolchildren. BMJ 1994;309:765-9.
2 Miltenburg D, Louw DF, Sutherland GR. Epidemiology of childhood brain tumors. Can J Neurol Sci 1996;23:118-22.

3 Vazquez-Barquero A, Ibanez FJ, Herrera S, et al. Isolated headache as the presenting clinical manifestation of intracranial tumors: a prospective study. Cephalalgia 1994;14:270-2.

4 Wöber-Bingöl C, Wöber C, Prayer D, et al. Magnetic resonance imaging for recurrent headache in childhood and adolescence. Headache 1996:36:83-90.

5 Lewis DL, Qureshi F. Acute headache in children and adolescents presenting to the emergency department. Headache 2000;40:200-3.

6 Lewis DW, Dorbad D. The utility of neuroimaging in the evaluation of children with migraine or chronic daily headache who have normal neurological examination. Headache 2000;40:629-32.

7 Maytal J, Bienkowski RS, Patel M, et al. The value of brain imaging in children with headache. Pediatrics 1995;96:413-16.

8 Whitehouse W. Specific causes of headache. In: Abu-Arafeh I, eds. Childhood headache. London: Mac Keith Press, 2002.

9 Abu-Arafeh I, Callaghan M. Headache clinics for children. In: Abu-Arafeh I, eds. Childhood headache. London: Mac Keith Press, 2002.

10 Headache Classification Committee of International Headache Society. Classification and diagnostic criteria for headache disorders, cranial neuralgia, and facial pain. Cephalalgia 1988;8(suppl 7):1-96.

11 Gardner MJ. Statistics with confidence. London: BMJ Publications, 1989.

12 Jordan JE, Ramirez GF, Bradley WG, et al. Economic and outcomes assessment of magnetic resonance imaging in the evaluation of headache. J Natl Med Assoc 2000;92:573-8.

13 Wang HZ, Simonson TM, Greco WR, et al. Brain MR imaging in the evaluation of chronic headache in patients without other neurologic symptoms. Acad Radiol 2001;8:405-8.

14 Edgeworth J, Bullock P, Bailey A, et al. Why are brain tumours still being missed? Arch Dis Child 1996;74:148-51.

\section{Call for papers}

11 th European Forum on Quality Improvement in Health Care 26-28 April 2006, Prague, Czech Republic

Deadline 30 September 2005.

For further information and to submit online go to: www.quality.bmipg.com 